\title{
Intraductal carcinoma of the prostate on needle biopsy: histologic features and clinical significance
}

\author{
Charles C Guo ${ }^{1}$ and Jonathan I Epstein ${ }^{1,2,3}$ \\ ${ }^{1}$ Department of Pathology, The Johns Hopkins University School of Medicine, Baltimore, MD, USA; \\ ${ }^{2}$ Department of Urology, The Johns Hopkins University School of Medicine, Baltimore, MD, USA and \\ ${ }^{3}$ Department of Oncology, The Johns Hopkins University School of Medicine, Baltimore, MD, USA
}

\begin{abstract}
Intraductal carcinoma of the prostate (IDC-P) has been described in radical prostatectomies. However, there is limited information as to its histologic features and clinical significance when seen on prostate biopsy. A total of 27 cases of prostate biopsies with only IDC-P (ie no infiltrating cancer anywhere on the biopsy) were studied from the consult files of one of the authors. IDC-P was defined as malignant epithelial cells filling large acini and prostatic ducts, with preservation of basal cells forming either: (1) solid or dense cribriform patterns or; (2) loose cribriform or micropapillary patterns with either marked nuclear atypia (nuclear size $6 \times$ normal or larger) or comedonecrosis. The numbers of cores involved by IDC-P in the biopsies ranged from 1 to 7 , with $>1$ core involved in 17 cases. The architectural patterns of IDC-P were solid (12), dense cribriform (19), loose cribriform (17), and micropapillary (5). More than one pattern was present in 24 of 27 cases. The cytological features frequently observed in IDC-P were marked pleomorphism (18), non-focal comedonecrosis (22), and mitoses (20). Basal cells were observed on regular hematoxylin and eosin stained slides in 14 cases; in all the cases, basal cells were confirmed by immunohistochemical stains for high molecular weight cytokeratin $(n=25)$ and/ or p63 $(n=4)$. After the diagnosis of IDC-P on prostate biopsies, patients were treated by radical prostatectomy (6), radiation (7), hormone (5), combined radiation and hormone (1), or watchful waiting (2). The follow-up information was not available for six patients. The follow-up times ranged up to 4 years with an average of 2.1 years. In all six radical prostatectomy specimens, high-grade infiltrating carcinoma with Gleason score 8 or 9 was present with five cases also revealing prominent IDC-P. Non-focal extraprostatic extension of carcinoma was observed in five of the six prostatectomy cases with two cases also demonstrating vascular invasion. Three of 16 patients who did not receive radical prostatectomy developed bone metastases. Our study indicates that IDC-P on prostate biopsies is frequently associated with high-grade cancer and poor prognostic parameters at radical prostatectomy as well as potentially advanced disease following other therapies. These findings support prior studies that IDC-P represents an advanced stage of tumor progression with intraductal spread of tumor. Consideration should be given to treat patients with IDC-P on biopsy aggressively even in the absence of documented infiltrating cancer.

Modern Pathology (2006) 19, 1528-1535. doi:10.1038/modpathol.3800702; published online 15 September 2006
\end{abstract}

Keywords: prostate; prostate cancer; intraductal carcinoma; prognosis

Intraductal carcinoma of the prostate (IDC-P) has been proposed in radical prostatectomy specimens to describe an atypical glandular lesion that spans the entire lumen of prostatic ducts or acini while the normal architecture of ducts or acini is still maintained. ${ }^{1-5}$ It has been observed in radical prostatec-

Correspondence: Dr JI Epstein, MD, Department of Pathology, The Johns Hopkins Hospital, 401 N. Broadway St, Rm 2242, Baltimore, MD 21231, USA.

E-mail: jepstein@jhmi.edu

Received 17 April 2006; revised 17 August 2006; accepted 21 August 2006; published online 15 September 2006 tomies that the presence of IDC-P is associated with high Gleason score, large tumor volume, extraprostatic extension of carcinoma, presence of carcinoma at surgical margins, and accelerated disease progression. ${ }^{3-5}$ IDC-P, while generally uncommon in prostate biopsies, is increasingly seen in those with large tumor volumes where substantial areas of IDC-P are observed in nearly half of prostatectomies with tumors larger than $4 \mathrm{~cm}^{3}$. Although we have previously described this phenomenon on needle biopsies, its morphological features and clinical significance on prostate biopsies remain largely unknown. ${ }^{6}$ 


\section{Materials and methods}

Twenty-seven cases of prostate biopsies with IDC-P were identified from the consult files of one of the authors between 2000 and 2005. Original slides were obtained for all the 27 cases and rereviewed. In all the cases, only IDC-P was observed and none had associated infiltrating prostatic adenocarcinoma on any core in the entire case. Our definition of IDC-P on needle biopsy shown in Table 1 was derived to identify objective morphological criteria that either architecturally or cytologically clearly exceed those seen in high-grade prostatic intraepithelial neoplasia (HGPIN). The differential diagnosis between IDC-P and HGPIN is further expanded upon in the Discussion. A dense cribriform pattern was one where there were more solid than luminal areas. More than one duct showing comedonecrosis was considered non-focal necrosis. Immunohistochemistry with high molecular weight cytokeratin (HMWCK) or p63 was typically performed at the outside institution. Information regarding clinical presentation, treatment, and follow-up were obtained from the medical records or from the correspondence with outside physicians.

Table 1 Definition of IDC-P

Malignant epithelial cells filling large acini and prostatic ducts, with preservation of basal cells and:

\section{or}

Solid or dense cribriform pattern

- Loose cribriform or micropapillary pattern with either

$\bigcirc$ Marked nuclear atypia: nuclear size $6 \times$ normal or larger

Non-focal comedonecrosis

\section{Results}

\section{Morphology}

Of the 27 patients with IDC-P on prostate biopsies, the average age was 69.9 years old (range: 52-86). The numbers of cores involved by IDC-P in each case ranged from 1 to 7 , with more than one core involved in 17 cases. The amount of tissue involved by IDC-P per case averaged 39.0\% (range: 8-75\%).

IDC-P on prostate biopsy was characterized by malignant epithelial cells filling large acini or prostatic ducts with retention of segments of the basal cell layer. In some cases, the contour and branching architecture of prostatic ducts was still recognized (Figure 1). Marked pleomorphism with large hyperchromatic nuclei (six times larger than those in adjacent normal glandular cells) was often observed (18 cases) (Figure 2). Other features frequently observed in IDC-P included central comedonecrosis (22 cases) and mitoses (20 cases) (Figures 1 and 3 ). Cases where cells within the center of the glands showed a more benign appearance than those more peripherally located was observed in nine cases (Figure 4). Basal cells were observed on regular hematoxylin and eosin (H\&E) stained slides in 14 cases; in all the cases, basal cells were confirmed by immunohistochemical stains for HMWCK (25 cases) and/or p63 (4 cases).

The common architectural patterns observed in IDC-P included solid (12 cases), dense cribriform (19 cases), loose cribriform (17 cases), and micropapillary (5 cases). In the solid pattern, sheets of malignant epithelial cells filled large acini or prostatic ducts (Figure 5), frequently accompanied by other ducts showing focal glandular features (Figure 3). In the dense cribriform pattern, malignant epithelial cells formed closely packed cribriform structures with small rounded lumens, where solid areas predominated over luminal spaces
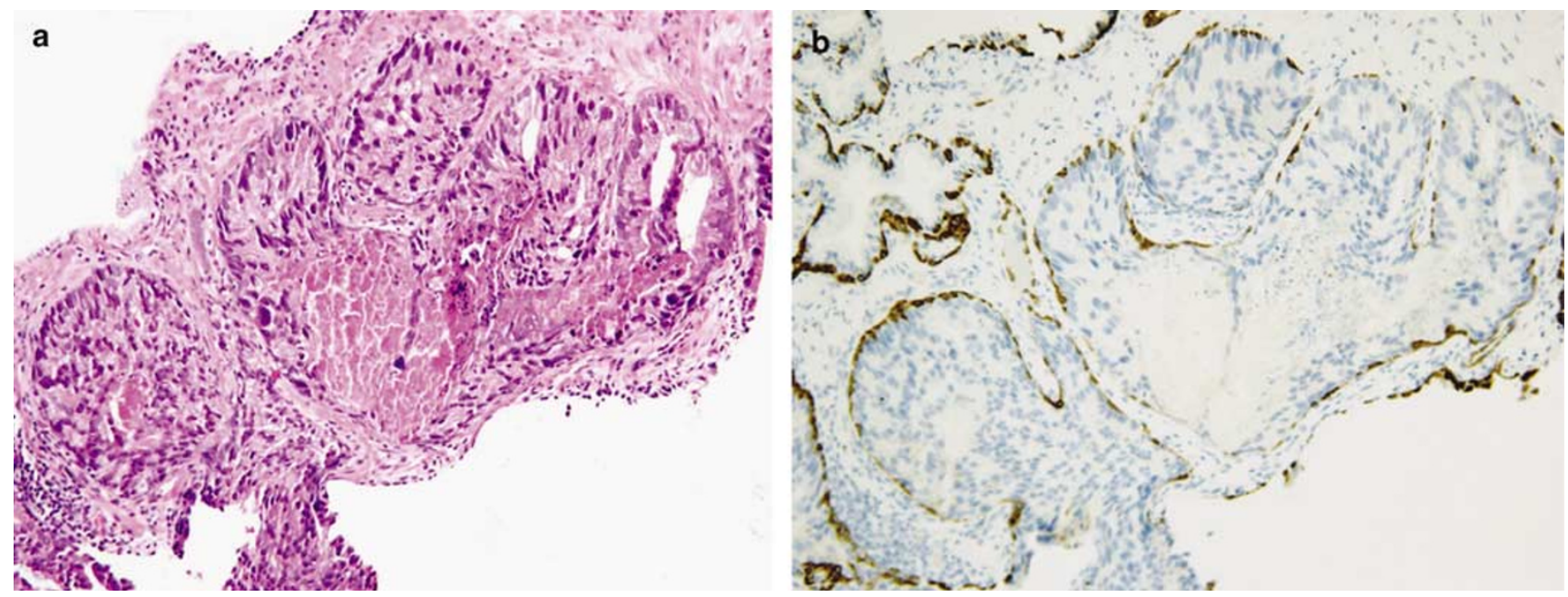

Figure 1 (a) Intraductal carcinoma of the prostate shows the contour and branching architecture of prostatic ducts with central coagulative necrosis. (b) HMWCK highlights basal cells around the prostatic ducts. 

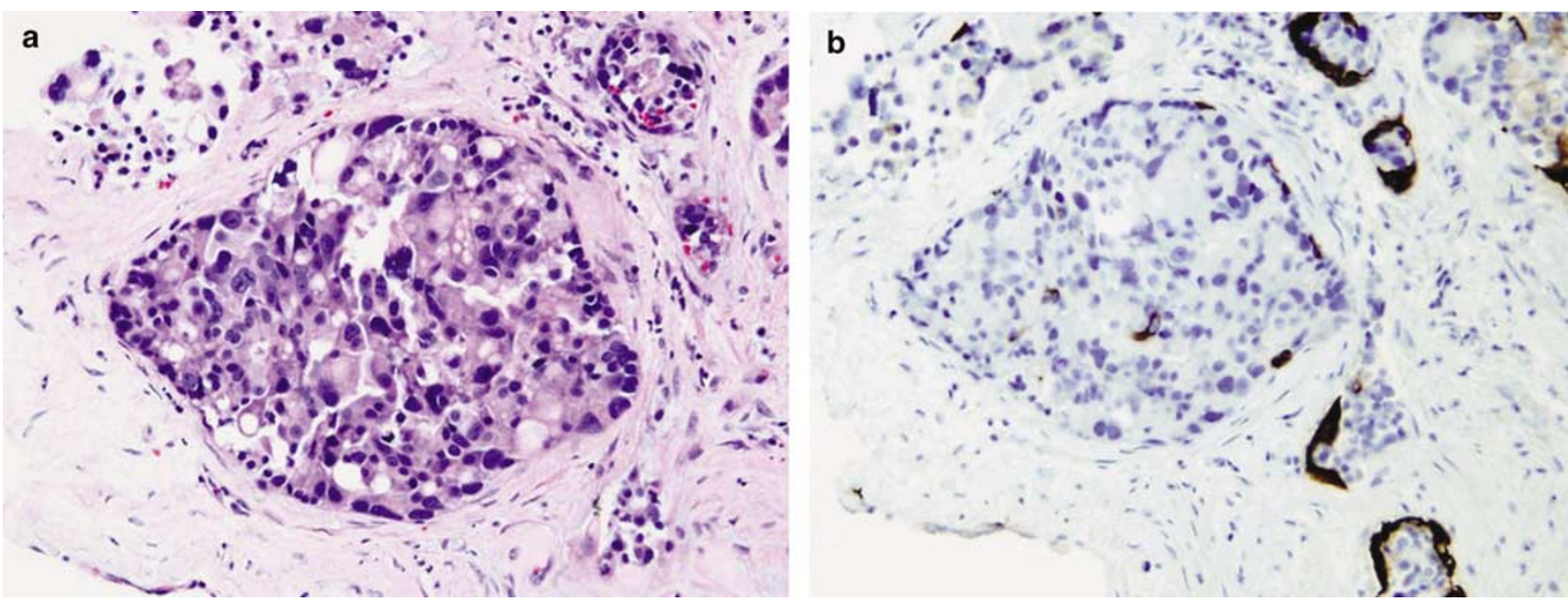

Figure 2 (a) Intraductal carcinoma of the prostate shows marked cellular atypia. (b) HMWCK highlights basal cells around the prostatic ducts.
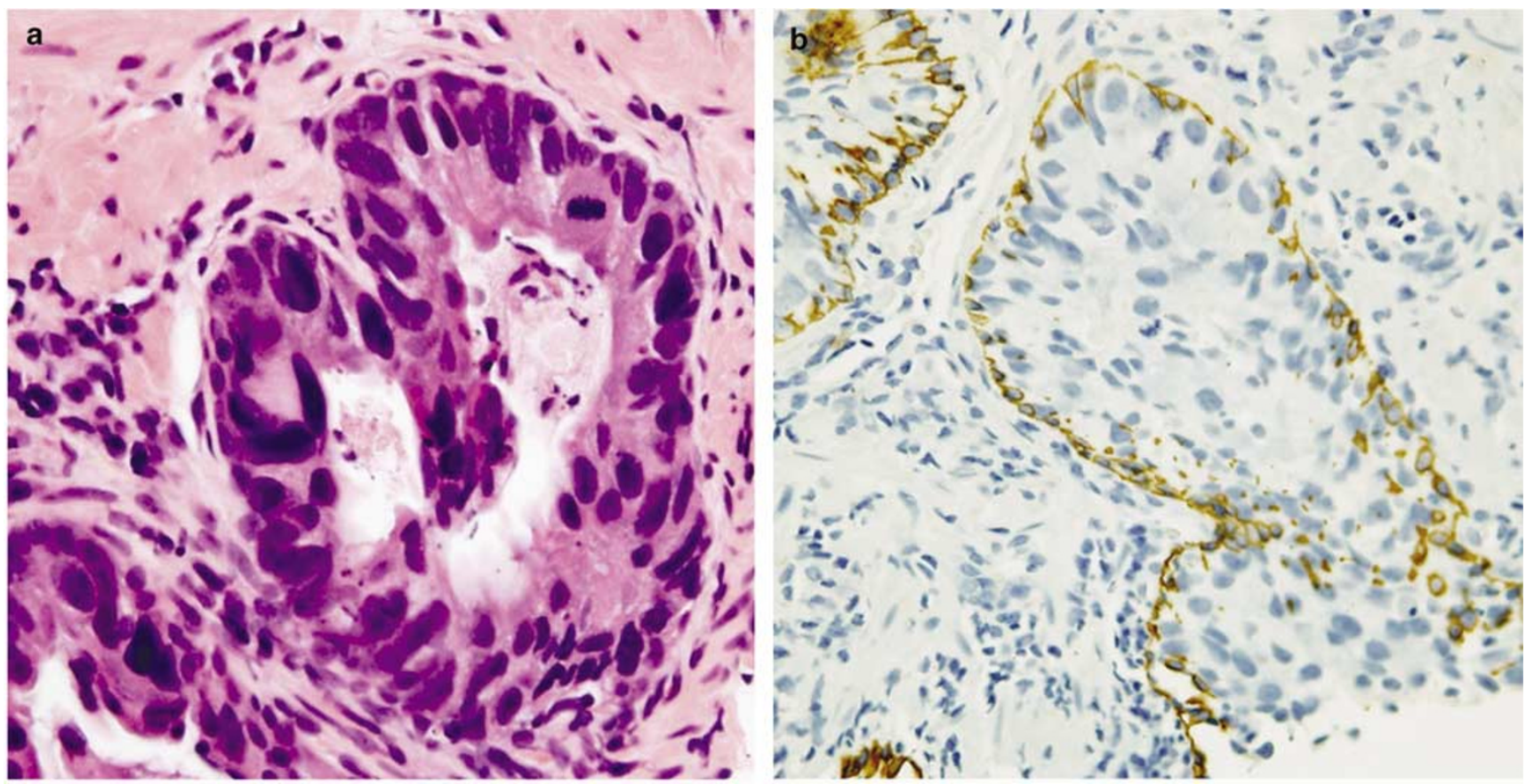

Figure 3 (a) Intraductal carcinoma of the prostate shows glandular feature with mitosis and marked nuclear atypia. (b) HMWCK highlights basal cells around the prostatic ducts.

(Figure 6). In contrast, the loose cribriform pattern contained larger lumina with relatively less epithelium (Figure 7). The micropapillary pattern was composed of malignant epithelial cells forming tufts or papillary structures in large acini or prostatic ducts (Figure 8). In 24 of the 27 cases (88.9\%), more than one pattern was observed.

\section{Treatment and Prognosis}

After diagnosis of IDC-P, patients were treated by radical prostatectomy (6), radiation (7), hormone (5), combined radiation and hormone (1) or watchful waiting (2). No follow-up information was available for six patients. Patients were followed for progression of disease, with follow-up periods ranging from 6 months to 4 years with an average of 2.1 years.

In the six patients who received radical prostatectomy, high-grade infiltrating cancer with Gleason score 8 or 9 was observed in the prostatectomy specimen in all six cases, while prominent IDC-P was also present in five cases (Figure 9). Non-focal extraprostatic extension of carcinoma was observed in five cases. Vascular invasion by carcinoma cells was seen in two cases including the case that did not 
have extraprostatic extension of carcinoma. On the initial prostate biopsies from these six patients, four demonstrated dense cribriform and solid patterns, and two showed loose cribriform and micropapillary patterns with comedonecrosis, mitoses and pleomorphism.

Of the 15 patients who did not receive radical prostatectomy, three patients developed bone metastases 6, 36, and 48 months after the diagnosis of IDC-P. Of the three patients, all had dense cribriform and solid patterns of IDC-P on their initial biopsies, and two patients also had extensive comedonecrosis and frequent mitoses. All of these three patients had been under hormonal therapy.

\section{Discussion}

The spread of prostatic acinar adenocarcinoma within adjacent benign prostatic ducts was first

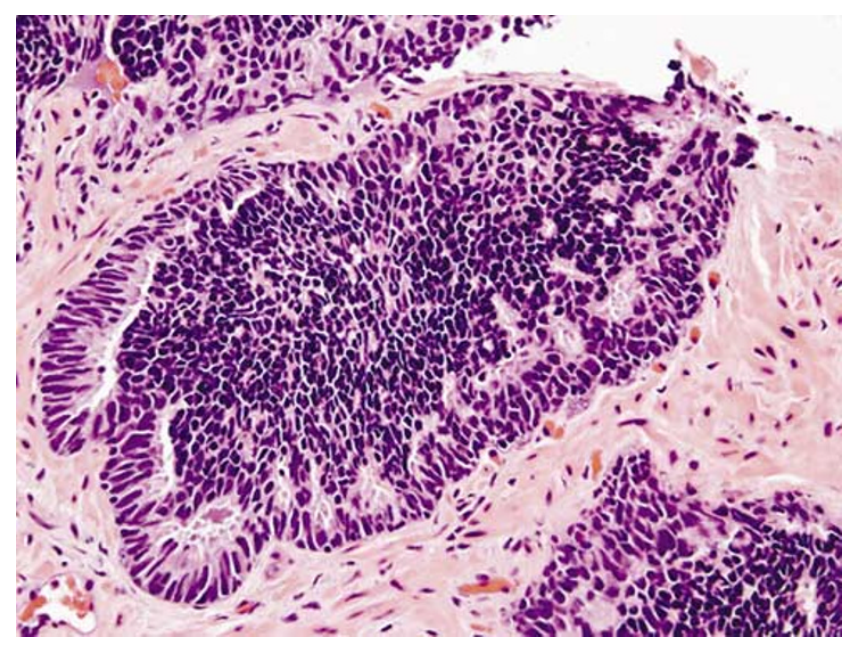

Figure 4 Intraductal carcinoma of the prostate shows smaller nuclei at the center of the cribriform than those more peripherally located. The cells form a sheet at the center, representing a solid pattern of IDC-P. reported by Kovi et $a l^{7}$ in a study of 139 cases of prostatic adenocarcinoma. In $48 \%$ of their cases, they observed that prostatic carcinoma cells penetrated the wall of benign ducts and progressively replaced the normal epithelial elements while the general framework of the affected duct appeared to be preserved. McNeal et al found that intraductal spread was particularly common in cribriform adenocarcinoma of the prostate ${ }^{2}$ and coined the term 'intraductal carcinoma of the prostate' to describe an unusual cribriform lesion characteristic of malignant glandular epithelial cells filling the lumina of prostatic ducts or acini. ${ }^{3}$ In a study of 130 radical prostatectomies, they found a strong association of IDC-P with high Gleason score, large tumor volume, positive surgical margins, and extensive perineural invasion, all of which contributed to an increased risk of progression following prostatectomy. IDC-P was an independently significant variable in the prediction of pathological stage, tumor volume, and treatment failure. ${ }^{8}$ The frequency of IDC-P increases sharply with increasing cancer volume. In the study by McNeal and Yemoto, ${ }^{3}$ IDC$\mathrm{P}$ was observed in $10 \%$ of cases with tumor volume less than $2 \mathrm{~cm}^{3}, 28 \%$ in tumors between 2 and $4 \mathrm{~cm}^{3}$, and $47 \%$ in tumors larger than $4 \mathrm{~cm}^{3}$. Wilcox et $a l^{5}$ observed IDC-P in $54 \%$ of cases with tumors larger than $4 \mathrm{~cm}^{3}$.

In several previous studies, the clinical outcome of IDC-P at prostatectomy appeared to be associated with certain histologic patterns. ${ }^{8,5}$ In the study by Cohen et $a l,{ }^{8}$ only one of six patients with trabecular (equivalent to micropapillary in our study) IDC-P progressed biochemically by 3 years, but 12 of 18 patients with solid and cribriform IDC-P had clinical or biochemical evidence of tumor progression. Wilcox et $a l^{5}$ demonstrated that cribriform IDC-P had a better prognosis than solid comedonecrosis IDC-P. In their study, the disease-free progression rate at 5 years was $78 \%$ for patients without IDC-P, decreasing to $65 \%$ for patients with cribriform IDC-P
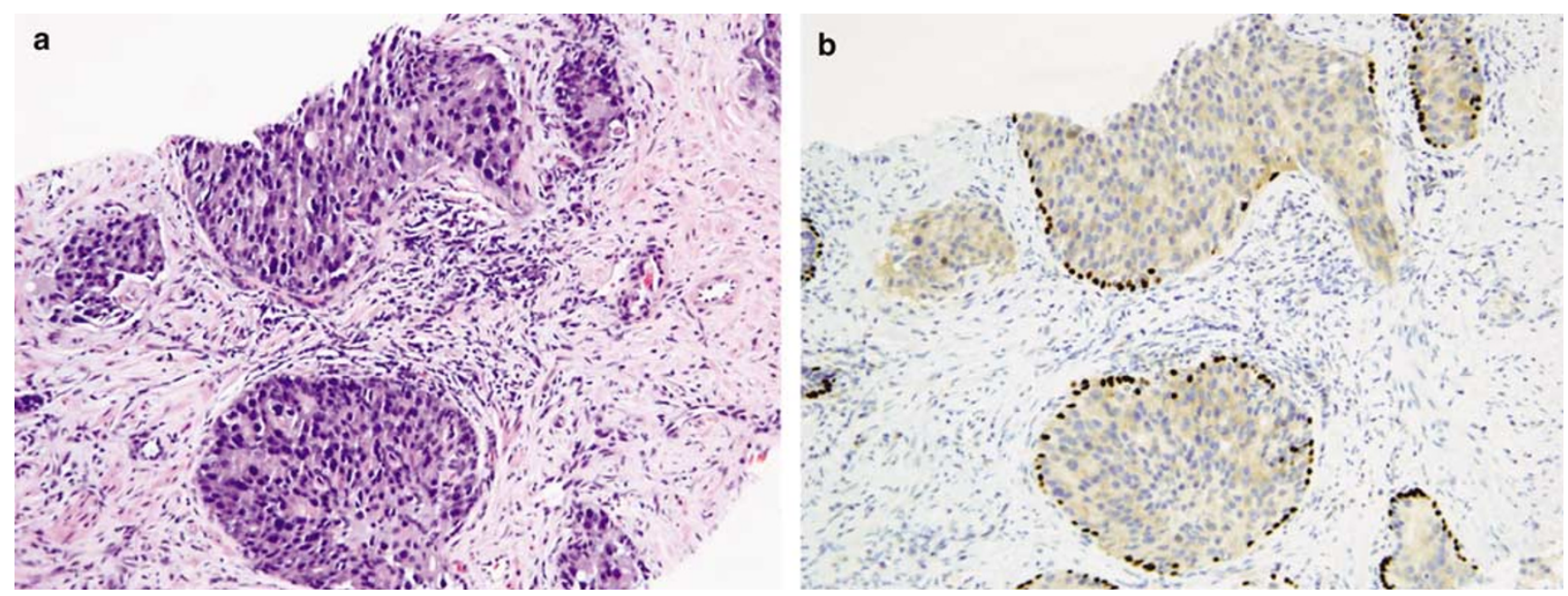

Figure 5 (a) Intraductal carcinoma of the prostate exhibits solid pattern. (b) p63 highlights basal cells around the prostatic ducts. 

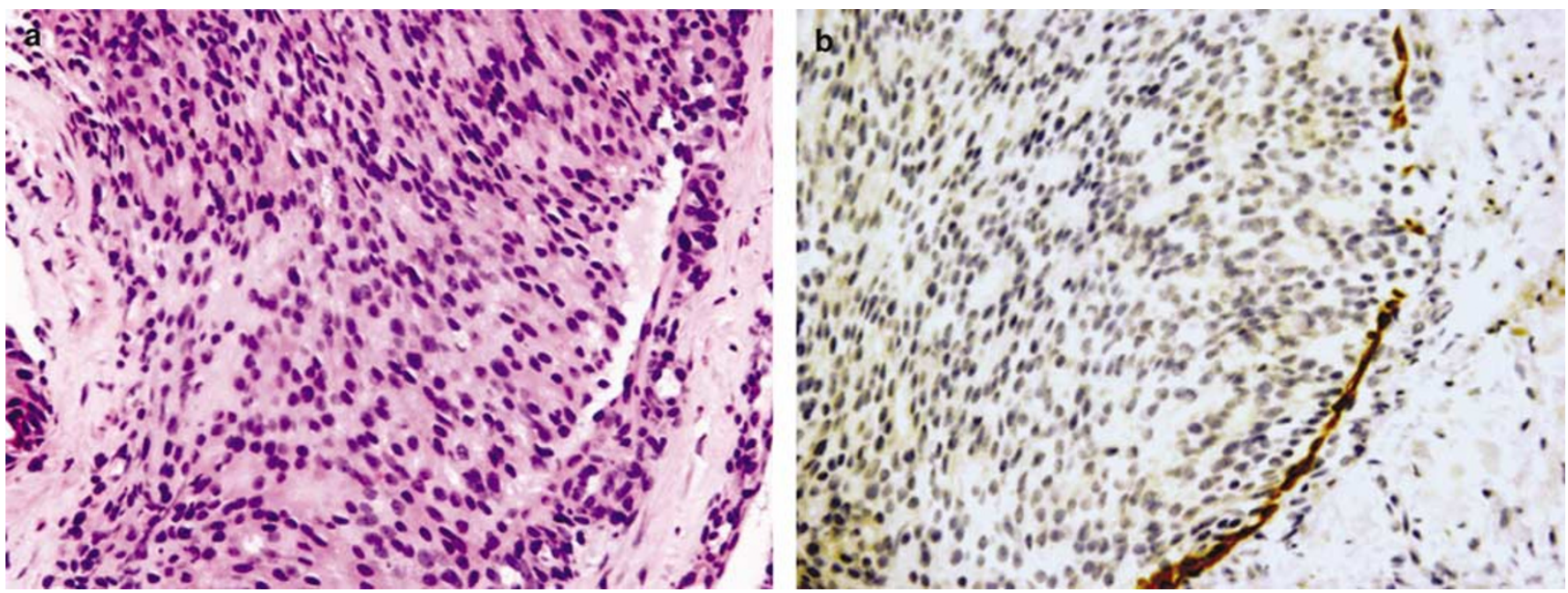

Figure 6 (a) Intraductal carcinoma of the prostate exhibits dense cribriform pattern with small rounded lumens. (b) HMWCK highlights basal cells around the prostatic ducts.
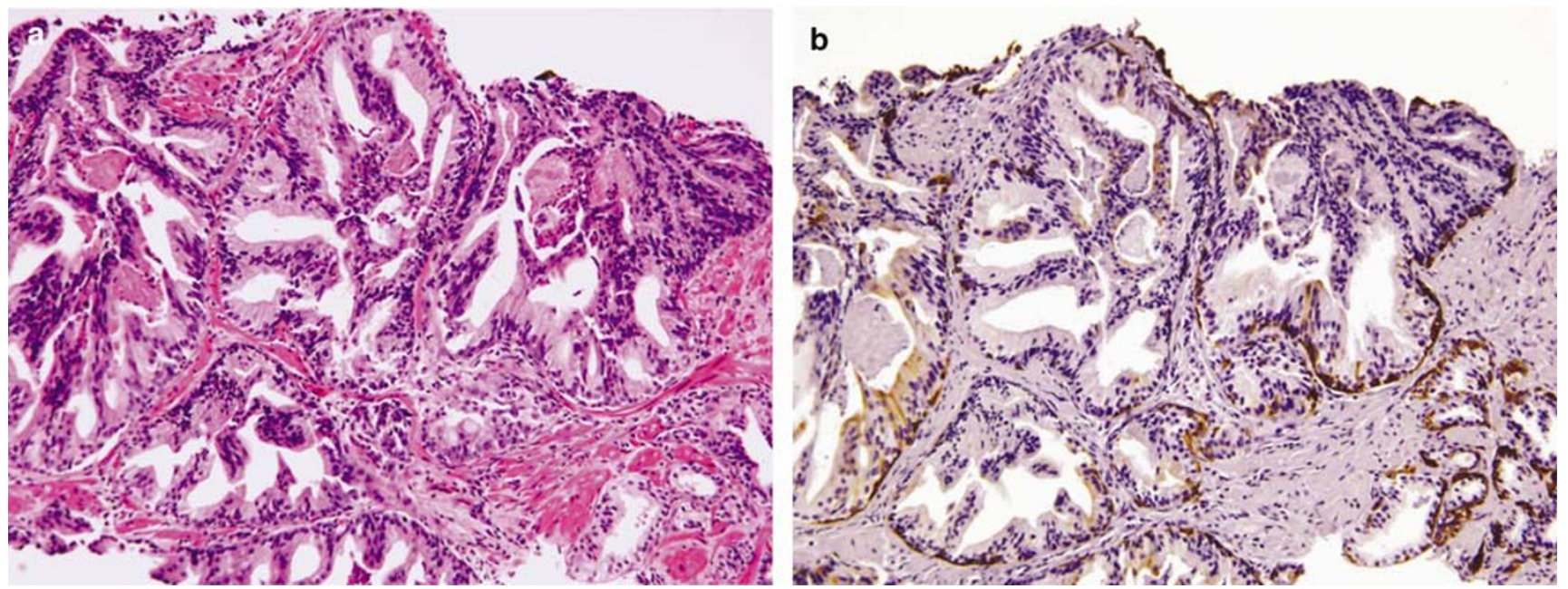

Figure 7 (a) Intraductal carcinoma of the prostate shows loose cribriform pattern with large irregular lumens. Central coagulative necrosis is also present. (b) HMWCK highlights basal cells around the prostatic ducts.

and only $20 \%$ for with solid and conmedonecrosis IDC-P. In our study, a relatively short follow-up period (2.1 years) revealed that metastases to bone developed in three of 15 patients who did not receive radical prostatectomy. In the prostate biopsies from all the three patients, the predominant patterns were solid and dense cribriform patterns. Of the six patients who received radical prostatectomy, two exhibited vascular invasion by carcinoma cells. The biopsies from these two patients also showed solid and dense cribriform as the predominant patterns.

IDC-P in the absence of infiltrating cancer is rare on biopsy. The 27 cases reported herein is out of a denominator of approximately 45000 prostate needle biopsy consults seen over the same 5 year period of time. While it may be argued that the incidence may even be lower as our series reflects the selection bias of consult cases, undoubtedly many cases of
IDC-P have been diagnosed as infiltrating high-grade prostate cancer in the absence of immunohistochemical stains for basal cells. The differential diagnosis of IDC-P on biopsy includes infiltrating cribriform acinar adenocarcinoma, ductal adenocarcinoma, intraductal spread of urothelial carcinoma, and HGPIN.

Infiltrating cribriform acinar adenocarcinoma (Gleason pattern 4 or Gleason pattern 5 with comedonecrosis) closely mimics cribriform IDC-P (Table 2). Many of the cases sent in consultation would have been diagnosed as such if immunohistochemistry demonstrating basal cells had not been performed at the outside institution. In some cases, the contour and branching pattern of normal duct architecture distinguishes IDC-P. Ultimately, the presence of a basal cell layer either identified on routine $\mathrm{H} \& \mathrm{E}$ prepared slides or with immunohistochemistry using HMWCK or p63 rules out infiltrat- 

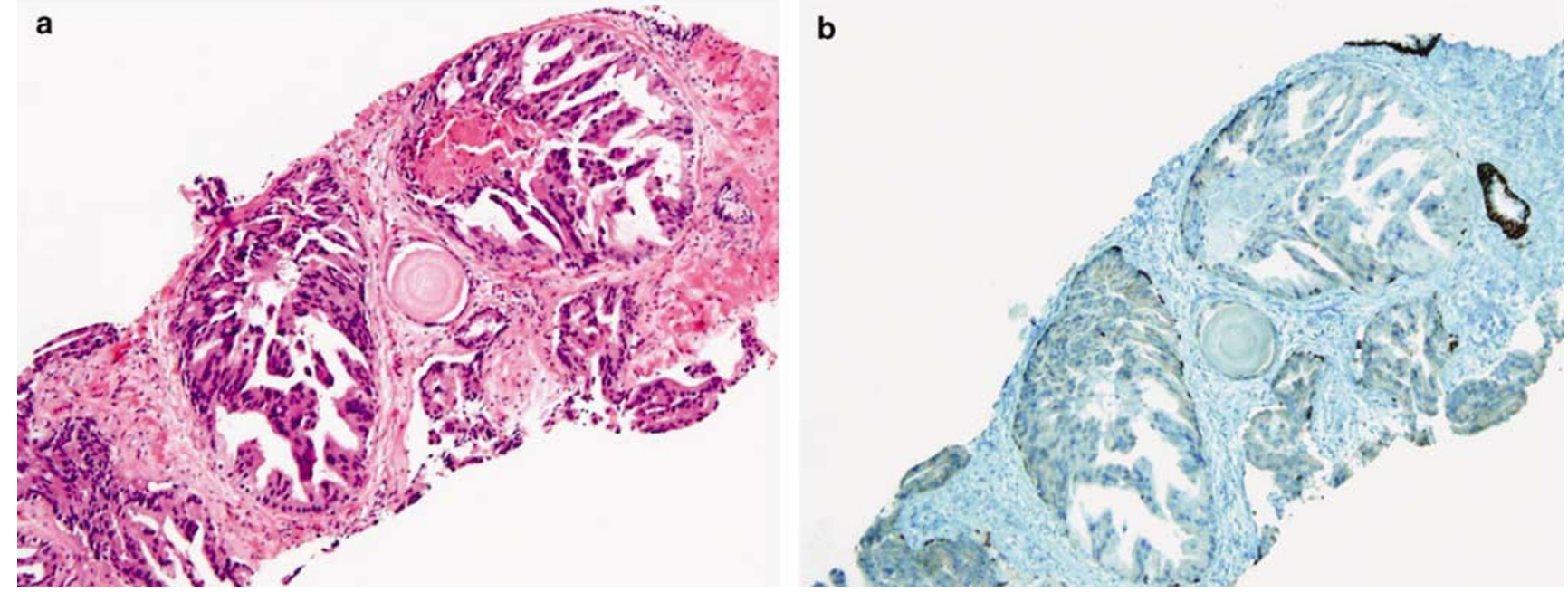

Figure 8 (a) Intraductal carcinoma of the prostate exhibits micropapillary pattern with central necrosis. (b) HMWCK highlights basal cells around the prostatic ducts.
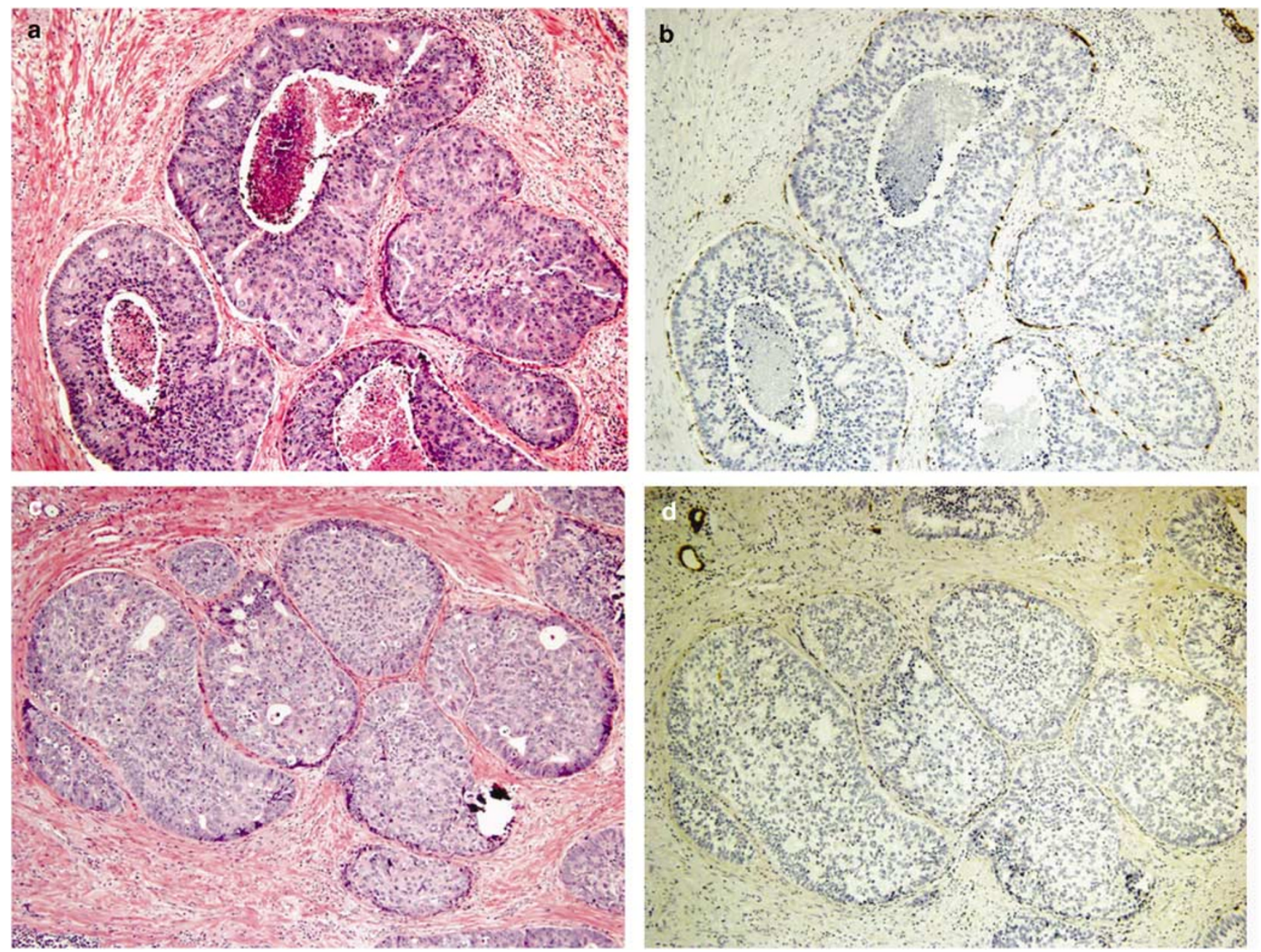

Figure 9 (a) In a radical prostatectomy following the diagnosis of IDC-P on prostate biopsy, a solid and dense cribriform pattern of IDC-P with prominent central necrosis. (b) HMWCK highlights basal cells around the prostatic ducts in IDC-P. (c) Adenocarcinoma of the prostate (Gleason score $5+4=9$ ) is also present. (d) HMWCK highlights the absence of basal cells in the periphery of the malignant glands. 
ing acinar prostate adenocarcinoma. The presence of comedonecrosis as a defining feature of Gleason pattern 5 adenocarcinoma assumes that the lesion is infiltrating cancer without a basal cell layer. Although there are extremely rare cases of early small foci of non-cribriform carcinoma of the prostate with focal retention of basal cell layer, ${ }^{9}$ this has never been described in cribriform, solid, or micropapillary prostate carcinoma.

There is significant morphological overlap between ductal adenocarcinoma of the prostate and IDC-P (Table 3). Distinctive features seen in ductal adenocarcinoma include tall pseudostratified columnar epithelium usually with amphophilic cytoplasm, classically arranged in cribriform patterns with slit-like spaces and/or true papillary fronds. ${ }^{10}$ In contrast, lesions we have designated as IDC-P have cuboidal cells, cribriform patterns with

Table 2 Cribriform acinar adenocarcinoma vs cribriform IDC-P

Cribriform acinar Cribriform IDC-P

adenocarcinoma

Absence of contour or branching architecture of prostatic ducts

Irregular, infiltrating borders Absence of basal cells

Table 3 Ductal adenocarcinoma vs IDC-P

\begin{tabular}{ll}
\hline Ductal adenocarcinoma & IDC-P \\
\hline Cribriform with large slit-like & Cribriform with small rounded \\
lumina & lumens \\
Tall columnar cells & Cuboidal cells \\
Papillary fronds with & Micropapillary tufts lacking \\
fibrovascular cores & fibrovascular cores \\
Basal cells usually absent & Basal cells always present \\
\hline
\end{tabular}

rounded lumina, and micropapillary tufts without fibrovascular cores. In addition, basal cells are generally absent in ductal adenocarcinoma, although occasionally there may be partial retention of basal cells as ductal adenocarcinoma can also spread within prostatic ducts. In cases where ductal adenocarcinoma is found within prostatic ducts and acini, it represents the analogous situation as IDC-P, which is acinar carcinoma found within prostatic ducts and acini. The concept and recognition of ductal adenocarcinoma is less controversial that IDC-P, as ductal adenocarcinoma has unique morphological features that in most cases help to distinguish it from HGPIN. However, in some cases there is a blurring of the distinction between HGPIN and ductal adenocarcinoma, just as there is in some cases between HGPIN and IDC-P. In both of these instances, the report should reflect uncertainty as to the differential diagnosis with a strong recommendation for repeat biopsy to identify more definitive carcinoma.

Solid patterns of IDC-P may mimic intraductal spread of urothelial carcinoma in the prostate, as both demonstrate solid intraductal-acinar involvement (Table 4). ${ }^{11}$ Other overlapping morphological features included marked nuclear pleomorphism, frequent mitotic activity, and conmedo-necrosis. However, solid patterns of IDC-P are often associated with cribriform or glandular patterns. When it is difficult to distinguish IDC-P from urothelial carcinoma, immunohistochemical studies generally resolve the problem as IDC-P is usually positive for PSA and PSAP, but negative for HMWCK and thrombomodulin, opposite to what is typically seen with urothelial carcinoma.

The most critical distinction is between HGPIN and IDC-P, as the former is typically not treated with definitive therapy and recent data has questioned whether HGPIN on needle biopsy even requires immediate rebiopsy within the first year following its diagnosis (Table 5). ${ }^{12}$ It has been questioned

Table 4 Intraductal spread of urothelial carcinoma (UC) vs IDC-P

Intraductal spread of UC IDC-P

Rarely associated with glandular features or cribriform pattern Immunohistochemically negative for PSA or PSAP

Positive for HMWCK or thrombomodulin
Often associated with focal glandular features and cribriform pattern Immunohistochemically positive for PSA or PSAP

Negative for HMWCK or thrombomodulin

Table 5 High-grade PIN vs IDC-P

\begin{tabular}{ll}
\hline High-grade PIN & IDC-P \\
\hline Lacks solid or dense cribriform patterns & Frequent solid or dense cribriform patterns \\
Enlarged nuclei (nuclear size 2-3 times those in normal cells) & Markedly enlarged nuclei (6 times larger than those in normal cells) \\
More uniform atypia between cells & May show marked pleomorphism \\
Glands rarely contain mitotic figures & Ducts/acini frequently have mitotic figures \\
Rare comedonecrosis & Frequent comedonecrosis
\end{tabular}


whether reproducible criteria can be developed to distinguish IDC-P from HGPIN. ${ }^{5}$ Both entities share cytologic features such nuclear enlargement, hyperchromasia, and enlarged nucleoli. Although the solid and dense cribriform patterns are not architectural patterns associated with HGPIN, loose cribriform and micropapillary patterns overlap between the two entities. To establish the diagnosis of IDC-P in the latter two patterns, other cytologic features such as markedly enlarged nuclei (six times larger than those in adjacent non-neoplastic cells) and non-focal comedonecrosis are required. Whereas it has been accepted that classic HGPIN can contain a rare gland with focal necrosis, more extensive necrosis is not acceptable. IDC-P also tends to show more prominent nuclear pleomorphism, as opposed to typical HGPIN with its uniformly enlarged nuclei. In cases where it is difficult to determine whether the cribriform structures are sufficiently dense to warrant a diagnosis of IDC-P, the presence of other features such as markedly enlarged nuclei or comedonecrosis can help to establish its diagnosis. Cases that do not satisfy the strict criteria proposed herein for IDC-P on needle biopsy yet appear more atypical either architecturally or cytologically than typical HGPIN can be diagnosed as borderline between IDC-P and HGPIN with a strong recommendation for repeat biopsy.

Despite its morphology resembling HGPIN, IDC-P is not likely a preinvasive neoplastic condition. While HGPIN is often present in prostate glands that have not yet developed invasive carcinoma, IDC-P is almost always associated with invasive cancer. Dawkins et $a l^{13}$ studied allelic instability in prostate cancers to define the position of IDC-P in the evolution sequence of prostate cancer progression. They found that $29 \%$ of Gleason pattern 4 cancers and $60 \%$ of IDC-P demonstrated loss of heterozygosity (LOH) while $\mathrm{LOH}$ was rarely observed in 'dysplasia' and Gleason pattern 3 adenocarcinoma. Their results indicate that IDC-P is a distinct lesion from HGPIN and represents a late event in prostate cancer evolution. Prostate cancer patients with IDC$\mathrm{P}$ also have a higher Gleason score and larger tumor volume and are more likely to show seminal vesicle involvement and disease progression than those without IDC-P. ${ }^{5}$ These findings support that IDC-P represents intraductal spread of carcinoma within pre-existing ducts and acini and should not be categorized as a preinvasive neoplastic condition.

In summary, IDC-P on needle biopsy is frequently associated with high-grade cancer and poor prognostic parameters at radical prostatectomy as well as potentially advanced disease following other therapies. These findings support prior studies that IDC-P represents an advanced stage of tumor progression with intraductal spread of tumor. Consideration should be given to treat patients with IDC-P on biopsy aggressively even in the absence of documented infiltrating cancer.

\section{References}

1 Cohen RJ, Chan WC, Edgar SG, et al. Prediction of pathological stage and clinical outcome in prostate cancer: an improved pre-operative model incorporating biopsy-determined intraductal carcinoma. Br J Urol 1998;81:413-418.

2 McNeal JE, Reese JH, Redwine EA, et al. Cribriform adenocarcinoma of the prostate. Cancer 1986;58:17141719.

3 McNeal JE, Yemoto CE. Spread of adenocarcinoma within prostatic ducts and acini. Morphologic and clinical correlations. Am J Surg Pathol 1996;20: 802-814.

4 Rubin MA, de La Taille A, Bagiella E, et al. Cribriform carcinoma of the prostate and cribriform prostatic intraepithelial neoplasia: incidence and clinical implications. Am J Surg Pathol 1998;22:840-848.

5 Wilcox G, Soh S, Chakraborty S, et al. Patterns of highgrade prostatic intraepithelial neoplasia associated with clinically aggressive prostate cancer. Hum Pathol 1998;29:1119-1123.

6 Epstein JI, Yang XM. Prostate Biopsy Interpretation, 3rd edn. Lippincott Williams \& Wilkins: Philadelphia, PA, 2002, pp 56-61.

7 Kovi J, Jackson MA, Heshmat MY. Ductal spread in prostatic carcinoma. Cancer 1985;56:1566-1573.

8 Cohen RJ, McNeal JE, Baillie T. Patterns of differentiation and proliferation in intraductal carcinoma of the prostate: significance for cancer progression. Prostate 2000;43:11-19.

9 Oliai BR, Kahane H, Epstein JI. Can basal cells be seen in adenocarcinoma of the prostate? An immunohistochemical study using high molecular weight cytokeratin (clone 34betaE12) antibody. Am J Surg Pathol 2002;26:1151-1160.

10 Epstein JI, Woodruff JM. Adenocarcinoma of the prostate with endometrioid features. A light microscopic and immunohistochemical study of ten cases. Cancer 1986;57:111-119.

11 Oliai BR, Kahane H, Epstein JI. A clinicopathologic analysis of urothelial carcinomas diagnosed on prostate needle biopsy. Am J Surg Pathol 2001;25:794-801.

12 Epstein JI, Herawi M. Prostate needle biopsies containing prostatic intraepithelial neoplasia or atypical foci suspicious for carcinoma: implications for patient care. J Urol 2006;175:820-834.

13 Dawkins HJ, Sellner LN, Turbett GR, et al. Distinction between intraductal carcinoma of the prostate (IDC-P), high-grade dysplasia (PIN), and invasive prostatic adenocarcinoma, using molecular markers of cancer progression. Prostate 2000;44:265-270. 